\title{
Critérios para a construção de jogos sérios
}

\author{
Rafaela Vilela da Rocha \\ Centro de Matemática, Computação e Cognição (CMCC) - Universidade Federal do \\ ABC (UFABC) - Santo André - SP - Brasil \\ rafaela.vilela@gmail.com
}

\begin{abstract}
This paper describes the requirements to support the balance among content, simulation, game and assessment, in the serious games development. From a literature review, concepts and theories were selected that support the creation of effective serious games. They were systematized and specified in 8 criteria, and grouped into: (a) scenarios and phases, (b) objectives, challenges, instructions and information, (c) interface, control, participation and autonomy, and (d) evaluation and feedback. Most of them were used in the creation of a serious game, which was used and evaluated by experts and learners. The results corroborate that the planning was adequate and balanced; and what other criteria are relevant.
\end{abstract}

Resumo. Este artigo descreve critérios que apoiam o balanceamento entre conteúdo, simulação, jogo e avaliação, na construção de jogos sérios. A partir de uma revisão de literatura, foram selecionados conceitos e teorias que embasam a criação de jogos sérios efetivos. Eles foram sistematizados e especificados em 8 critérios, e agrupados em: (a) cenários e fases, (b) objetivos, desafios, instruções e informações, (c) interface, controle, participação e autonomia do aprendiz, e (d) avaliação e feedback. A maioria deles foi utilizada na criação de um jogo sério, o qual foi usado e avaliado por especialistas e aprendizes. Os resultados corroboram que o planejamento foi adequado e balanceado; e que os demais critérios seriam relevantes.

\section{Introdução}

Jogos sérios são jogos usados para fins de aprendizagem e de treinamento de pessoas, tais como, nas áreas de meio ambiente, saúde, defesa [Marklund, 2013]. Eles vêm sendo criados e utilizados em diversos contextos educacionais, porém, seu desenvolvimento é um processo complexo, com alto custo (de recursos humanos, materiais, financeiros, espaço e tempo). Rocha, Bittencourt e Isotani (2014) discutem sete desafios relacionados às: (1) características do produto final - que requer balanceamento de aspectos pedagógicos (conteúdo, avaliação, feedback) e de jogabilidade (desafio, controle, imersão); (2) inclusão de competências a serem ensinadas, treinadas e avaliadas; (3) integração de diferentes profissionais em sua construção; (4) sistematização e padronização de artefatos e processos em seu desenvolvimento; (5) reuso e extensão desses artefatos; (6) avaliação e (7) validação, tanto do aprendizado/treinamento quanto do jogo sério. Para oferecer suporte para a construção de jogos sérios, superando estes desafios, diferentes metodologias emergiram, além das existentes nas áreas inter-relacionadas. Entretanto, de forma geral, essas metodologias não apoiam a construção do jogo sério de forma a compreender seus múltiplos 
VI Congresso Brasileiro de Informática na Educação (CBIE 2017)

Anais do XXVIII Simpósio Brasileiro de Informática na Educação (SBIE 2017)

requisitos, a partir de conceitos e teorias relacionadas. Dessa forma, muitas vezes há um desbalanceamento, principalmente entre jogabilidade e conteúdo. Isso pode desmotivar e desengajar o aprendiz a jogar, ou não ser eficiente quanto ao seu objetivo de aprendizado, treinamento e avaliação [Marklund, 2013].

Neste contexto, este artigo descreve um conjunto critérios, os quais visam apoiar a adequação, integração e o balanceamento entre conteúdo, simulação, jogo, treinamento, aprendizagem e avaliação, a partir de teorias e trabalhos relacionados. Na seção 2, é apresentado o estado da arte de metodologias de desenvolvimento. Na seção 3 , é apresentada a fundamentação teórica. $\mathrm{Na}$ seção 4, os critérios são especificados. $\mathrm{Na}$ seção 5, são apresentados os resultados da criação de um jogo sério usando esses critérios, seguidas das discussões (na seção 6) e considerações finais (na seção 7).

\section{Trabalhos Relacionados}

Uma revisão da literatura foi realizada para obter uma compreensão geral do estado da arte e as limitações das metodologias existentes para criação de jogos sérios. Foram pesquisados artigos nos principais motores de busca, em congressos e periódicos; livros e sites de referência que descrevem metodologias de desenvolvimento de jogos sérios e produtos relacionados; além de consultadas as referências citadas nesses trabalhos.

Elas podem ser agrupadas em metodologias de: (1) Design de jogos de entretenimento [Chandler, 2012; Novak, 2010; Schuytema, 2008]; (2) Modelagem de simulações [Balci, 2012; Banks et al., 2001; IEEE, 2003]; (3) Design de instruções e treinamentos [ABNT, 2001; Dick, Carey e Carey, 2004]; (4) Design de simulações e jogos educacionais [Adams et al, 2007; Aslan e Balci, 2015; Hays, 2005]; e (5) Desenvolvimento de jogos sérios [Becker e Parker, 2012; Freitas e Jarvis, 2006; Kirkley, Tomblin e Kirkley, 2005], conforme descritas e analisadas em [Rocha, 2014].

De forma geral, nas metodologias de desenvolvimento de jogos faltam detalhamentos/ documentações dos processos e dos artefatos produzidos; e faltam inclusões de requisitos de simulação, aprendizagem, treinamento e avaliação. Nas metodologias de modelagem e simulação: faltam detalhes dos requisitos instrucionais, de treinamento, de avaliação e do modelo de representação. Por outro lado, as especificações de design instrucional e de treinamento não abrangem requisitos de: jogos e simulações; e há diferenças significativas entre materiais educacionais e jogos sérios, tais como, linearidade, conteúdos estruturados e lógicos para auxiliar o instrutor, ênfase no processo em vez de foco no aprendizado e aprendizes. As metodologias de criação de simulações e jogos educacionais não oferecem apoio para diferentes avaliações e feedback. As metodologias de desenvolvimento de jogos sérios têm foco no design instrucional e do jogo, porém sem integração detalhada e especificada.

\section{Fundamentação Teórica}

Para construir um jogo sério efetivo, é necessária uma visão holística e multidisciplinar dos requisitos de jogo, simulação, aprendizagem, treinamento e avaliação com o domínio de aplicação [Rocha, 2014]. A partir de uma revisão de literatura, foram selecionados conceitos e teorias que embasam a construção de jogos sérios efetivos, ou seja, que proporcionam o aprendizado/ treinamento planejado, satisfazem e motivam o aprendiz, e apoiam o processo de avaliação e feedback; conforme descrito a seguir. 
VI Congresso Brasileiro de Informática na Educação (CBIE 2017)

Anais do XXVIII Simpósio Brasileiro de Informática na Educação (SBIE 2017)

\subsection{Simulações Educacionais e de Treinamento}

As simulações educacionais e de treinamento visam à modelagem do comportamento de um sistema ou processo, para fins de aprendizagem. Fidelidade é o grau com que o modelo de simulação representa o fenômeno, equipamento ou sistema do mundo real sendo simulado. A fidelidade pode ser física, funcional ou psicológica [1.1] [Feinstein e Cannon, 2002]. A fidelidade física é o grau em que o modelo de simulação se parece (visual, sonoro e tátil, a partir de sua interface e controles) com o equipamento, ambiente ou fenômeno real. A fidelidade funcional é o grau em que o modelo de simulação age (informações, estímulos e respostas) e reage às tarefas sendo executadas (comportamento e interação) comparado à realidade. A fidelidade psicológica é o grau em que o modelo de simulação provoca fatores psicológicos nos aprendizes comparado ao que acontece nas tarefas reais, tais como, estresse e medo [Alexander et al., 2005].

\subsection{Aprendizagem e Treinamento}

Foram feitas pesquisas nas áreas de teorias de design instrucional e de aprendizagem, programa de treinamento, medições, avaliações e feedback. A partir delas, foram selecionados requisitos para aprendizagem e treinamento eficientes, listados a seguir: [2.1] Elaboração do programa de treinamento com contexto significativo e objetivos claros das competências a partir da identificação das necessidades da instituição, conforme ABNT (2001); [2.2] Planejamento das atividades com progressão de dificuldade, conforme Taxonomia de Bloom (1956); [2.3] Inclusão de todos os perfis de aprendizes e suas estratégias de aprendizagem, da teoria de aprendizagem experiencial e estilo de aprendizagem de Kolb e Kolb (2005); [2.4] Inclusão de diversos tipos de avaliação do desempenho do aprendiz (diagnóstica, formativa, somativa, autoavaliação), conforme Miller, Imrie e Cox (1998) e Piskurich, Beckschi e Hall, (2000); [2.5] Medições e avaliações contínuas: do processo (o que o aprendiz está fazendo) e do resultado (final do jogo), conforme Salas et al. (2009); [2.6] Feedback imediato ao longo do treinamento e ao final (para reforçar os acertos e corrigir os erros), conforme Salas et al. (2009); [2.7] Inclusão de diferentes níveis de avaliação do programa de treinamento (principalmente, reação e aprendizado), conforme Teoria de Avaliação do Programa de Treinamento (TAPT), de Kirkpatrick e Kirkpatrick, (2006).

\subsection{Jogos}

Trybus (2014) apresenta seis princípios de aprendizagem efetiva, que geralmente os jogos contemplam: níveis, desafios, aprendizagem ativa, feedback imediato, prática das competências, motivação extrínseca e intrínseca, objetivos e pré-requisitos claros, vários cenários ou problemas [3.1]. Além disso, outros autores descrevem teorias e conceitos importantes para motivar e engajar os aprendizes, além de superar os desafios de falta de jogabilidade e balanceamento. Os requisitos definidos a partir da literatura incluem também: [3.1] Uso da teoria expectativa-valor de Keller (2009), para incluir estratégias motivacionais; [3.3] Abrangência da experiência de usuários em jogos [Novak, 2010]; [3.4] Balanceamento da jogabilidade (exploração da experiência do aprendiz, desafios, estratégias, interatividade com o conteúdo de aprendizagem e a simulação [Engström et al., 2011]; e (3.5) Uso da taxonomia de Bloom para definir os níveis e desafios do jogo [Bloom, 1956]. 
VI Congresso Brasileiro de Informática na Educação (CBIE 2017)

Anais do XXVIII Simpósio Brasileiro de Informática na Educação (SBIE 2017)

\subsection{Domínio de Aplicação}

Foram analisados conceitos e teorias do domínio de resposta a emergências, que são pertinentes também para outros contextos educacionais. Assim, os requisitos para criação de conteúdos adequados são: [4.1] Análise dos erros humanos, usando a teoria de dimensões de erros humanos (da área de engenharia cognitiva), de Hollnagel et al. (2011); [4.2] Uso de manuais e Procedimentos Operacionais Padrão (POPs); [4.3] Análise das competências que devem ser treinadas pelos profissionais; [4.4] Análise de como são criados os exercícios simulados reais e os treinamentos a distância.

\section{Critérios para Construção de Jogos Sérios}

Os conceitos e teorias importantes para a criação de jogos sérios efetivos, apresentados na seção anterior, foram sistematizados e organizados em oito critérios, que estão especificados em checklist e agrupados em subprodutos, conforme descritos a seguir.

\subsection{Cenários e Fases}

Jogos sérios são efetivos quando contêm cenários relevantes (criados a partir da realidade) e com diferentes formas de avaliação (não uma única em um cenário, mas formando um processo / programa de avaliação), com feedback ao longo do jogo e não somente ao final (Salas, 2009). Para isso, é necessário projetar diferentes cenários e fases em conjunto com as avaliações que serão feitas, levando em consideração o conhecimento prévio e os erros que podem ser realizados em cada situação (Quadro 1).

Quadro 1. Critérios para construção de cenários e fases do jogo sério.

\begin{tabular}{|c|c|c|}
\hline Critério & Checklist & Referências \\
\hline \multirow{3}{*}{$\begin{array}{l}1 . \\
\text { Relevância } \\
\text { e fidelidade } \\
\text { de cenários }\end{array}$} & $\begin{array}{l}\text { Criar cenários com exemplos concretos a partir da realidade, e } \\
\text { usando documentação existente (manuais, protocolos, etc.) }\end{array}$ & $\begin{array}{c}\text { Atenção [3.2], POPs } \\
{[4.2,4.4]}\end{array}$ \\
\hline & $\begin{array}{l}\text { Planejar o grau de fidelidade física, comportamental e } \\
\text { psicológica de acordo com as competências a serem treinadas. }\end{array}$ & Fidelidade [1.1] \\
\hline & Criar cenários explorando as dimensões de erros humanos. & Erros humanos [4.1] \\
\hline \multirow{11}{*}{$\begin{array}{c}2 . \\
\text { Variedade } \\
\text { de fases do } \\
\text { programa } \\
\text { de } \\
\text { treinamento }\end{array}$} & $\begin{array}{l}\text { Definir contexto significativo com objetivos claros das } \\
\text { competências que devem ser praticadas (conhecimentos, } \\
\text { habilidades e atitudes). }\end{array}$ & $\begin{array}{l}\text { Programa de treinamento } \\
\text { e competências treinadas } \\
{[2.1,4.3]}\end{array}$ \\
\hline & $\begin{array}{l}\text { Incluir vários formatos de conteúdos (p.ex. texto, imagem, } \\
\text { áudio, vídeo) e interações. }\end{array}$ & Atenção [3.2] \\
\hline & Incorporar os requisitos de aprendizagem. & Confiança [3.2] \\
\hline & Explorar a experiência prévia e interesses do jogador. & Relevância [3.2] \\
\hline & $\begin{array}{l}\text { Permitir que o jogador utilize a habilidade adquirida em um } \\
\text { novo cenário. }\end{array}$ & Satisfação [3.2] \\
\hline & Adaptar as fases ao nível de competências prévias individuais. & Níveis [3.1] \\
\hline & $\begin{array}{l}\text { Criar fases que garantam que o aprendiz pratique até que o } \\
\text { conhecimentos e habilidades se tornem automatizados e } \\
\text { consolidados na memória (domínio completo dos objetivos). }\end{array}$ & $\begin{array}{l}\text { Prática das competências } \\
\qquad[3.1]\end{array}$ \\
\hline & $\begin{array}{l}\text { Balancear conteúdo de aprendizagem, simulação e } \\
\text { jogabilidade. }\end{array}$ & $\begin{array}{c}\text { Equilíbrio do jogo- } \\
\text { conteúdo- simulação[3.4] }\end{array}$ \\
\hline & Incluir avaliação da reação do aprendiz. & Nível $1 \mathrm{da}$ TAPT [2.7] \\
\hline & Incluir avaliação do aprendizado/ desempenho do aprendiz. & Nível 2 da TAPT [2.7] \\
\hline & $\begin{array}{l}\text { Incluir diversos tipos de avaliação do desempenho do } \\
\text { aprendiz (diagnóstica, formativa, somática, autoavaliação). }\end{array}$ & $\begin{array}{c}\text { Avaliação de } \\
\text { desempenho [2.4] }\end{array}$ \\
\hline
\end{tabular}


VI Congresso Brasileiro de Informática na Educação (CBIE 2017)

Anais do XXVIII Simpósio Brasileiro de Informática na Educação (SBIE 2017)

\subsection{Objetivos, Desafios, Instruções e Informações}

Os objetivos de um jogo sério devem ser claros e terem desafios progressivos. As instruções apresentadas devem ser claras, relevantes e explicar os critérios de avaliação, conforme apresentado no Quadro 2.

Quadro 2. Critérios para a descrição de objetivos e instruções no jogo sério.

\begin{tabular}{|c|c|c|}
\hline Critério & Checklist & Referências \\
\hline \multirow{6}{*}{$\begin{array}{l}\text { 3. Clareza e } \\
\text { progressão dos } \\
\text { objetivos e } \\
\text { desafios }\end{array}$} & Incluir objetivos que são além às competências do jogador. & Desafio [3.2] \\
\hline & Incluir conflitos. & Atenção [3.2] \\
\hline & $\begin{array}{l}\text { Aumentar o nível de dificuldade dos desafios, com incremento gradual } \\
\text { para explorar motivação e experiência. }\end{array}$ & $\begin{array}{c}\text { Taxonomia de } \\
\text { Bloom }[2.2,3.5]\end{array}$ \\
\hline & Definir fases com objetivos claros e dificuldade incremental. & $\begin{array}{l}\text { Objetivos e pré- } \\
\text { requisitos [3.1] }\end{array}$ \\
\hline & $\begin{array}{l}\text { Progredir as fases conforme o ritmo de cada aprendiz (rápido ou } \\
\text { devagar, novato ou especialista). }\end{array}$ & Níveis [3.1] \\
\hline & $\begin{array}{l}\text { Reforçar a motivação intrínseca (razão pessoal em fazer a atividade), } \\
\text { como por exemplo, passar para níveis mais complexos pode aumentar } \\
\text { a autoestima e motivação devido a uma maior competência. }\end{array}$ & Motivação [3.1] \\
\hline \multirow{2}{*}{$\begin{array}{l}\text { 4. Relevância } \\
\text { e clareza de } \\
\text { instruções e } \\
\text { informações }\end{array}$} & $\begin{array}{l}\text { Apresentar os méritos do conteúdo a ser treinado/aprendido e } \\
\text { relacioná-los com objetivos futuros (pessoais e do próprio jogo). }\end{array}$ & Relevância [3.2] \\
\hline & Explicar os critérios de avaliação de desempenho. & Confiança [3.2] \\
\hline
\end{tabular}

\subsection{Interface, Controle, Participação e Autonomia do Aprendiz}

A interface e os controles do jogo permitem, se forem planejados adequadamente, que os jogadores interajam com o jogo de forma fácil e engajada, sejam independentes e ativos. Esses elementos são importantes para a aprendizagem significativa e satisfação dos aprendizes, que não serão meros expectadores ou participantes passivos. Dessa forma, é necessário que a interface e controle seja clara e simples, e que o jogo foque no engajamento e autonomia do aprendiz, conforme especificado no Quadro 3.

\section{Quadro 3. Critérios para as definições da interface, controle, exploração da} participação e independência do aprendiz.

\begin{tabular}{|c|c|c|}
\hline Critério & Checklist & Referências \\
\hline \multirow{3}{*}{$\begin{array}{l}\text { 5. Clareza e } \\
\text { simplicidade } \\
\text { na interface e } \\
\text { controle do } \\
\text { jogo }\end{array}$} & $\begin{array}{l}\text { Possibilitar fácil manipulação do jogo conforme as ações } \\
\text { intencionadas sobre a interface e com os controles do jogo. }\end{array}$ & Controle [3.3] \\
\hline & $\begin{array}{l}\text { Possibilitar que o jogador aprenda as habilidades necessárias (de } \\
\text { controle e interação) para explorar e atingir os objetivos do jogo. }\end{array}$ & Competência [3.3] \\
\hline & $\begin{array}{l}\text { Fornecer oportunidades de interações e escolhas para realizar um } \\
\text { objetivo. }\end{array}$ & Relevância [3.2] \\
\hline \multirow{3}{*}{$\begin{array}{l}\text { 6. } \\
\text { Participação e } \\
\text { independência } \\
\text { do aprendiz }\end{array}$} & $\begin{array}{l}\text { Criar tarefas práticas que exigirem aprendizagem ativa e explorem } \\
\text { aprendizagem experiencial e diversificada }\end{array}$ & $\begin{array}{l}\text { Aprendizagem } \\
\quad \text { ativa [3.1] e } \\
\text { significativa [2.3] }\end{array}$ \\
\hline & $\begin{array}{l}\text { Estimular a exploração e participação do jogador, bem como seu } \\
\text { envolvimento no jogo. }\end{array}$ & $\begin{array}{l}\text { Atenção [3.2], } \\
\text { Imersão [3.3] }\end{array}$ \\
\hline & $\begin{array}{l}\text { Possibilitar autoconfiança e independência no aprendizado e } \\
\text { prática, de modo que estes elementos possam impulsionar a } \\
\text { confiança e o progresso do aprendiz. }\end{array}$ & Confiança [3.2] \\
\hline
\end{tabular}

\subsection{Avaliação e Feedback}

O feedback imediato é essencial para que o aprendiz se sinta no controle do jogo, bem como, para recompensar os acertos e corrigir os erros. Entretanto, a coleta de dados, a 
VI Congresso Brasileiro de Informática na Educação (CBIE 2017)

Anais do XXVIII Simpósio Brasileiro de Informática na Educação (SBIE 2017)

avaliação e medição do desempenho, muitas vezes são negligenciadas em jogos sérios, o que pode afetar a motivação do aprendiz em jogar, ou a eficiência do jogo, visto que os erros realizados pelos aprendizes não enfatizados e corrigidos. Dessa forma, além da objetividade e amplitude da avaliação, já parcialmente descrita em outros critérios e compilada a seguir, é necessário a objetividade e amplitude de feedback (Quadro 4).

Quadro 4. Critérios para a elaboração de avaliações e feedback.

\begin{tabular}{|c|c|c|}
\hline Critério & Checklist & Referências \\
\hline \multirow{5}{*}{$\begin{array}{l}7 . \\
\text { Objetividade } \\
\text { e amplitude } \\
\text { da avaliação }\end{array}$} & $\begin{array}{l}\text { Incluir diversos tipos de avaliação do desempenho do aprendiz } \\
\text { (diagnóstica, formativa, somática, autoavaliação) (idem 2) }\end{array}$ & $\begin{array}{c}\text { Avaliação de } \\
\text { desempenho [2.4] }\end{array}$ \\
\hline & $\begin{array}{l}\text { Fornecer ferramentas de autoavaliação baseada em objetivos } \\
\text { bem definidos. }\end{array}$ & Confiança [3.2] \\
\hline & $\begin{array}{l}\text { Incluir medições e avaliações contínuas do processo (o que o } \\
\text { jogador está fazendo) e do resultado (final do jogo) }\end{array}$ & $\begin{array}{l}\text { Sistema de medição } \\
\text { de desempenho [2.5] }\end{array}$ \\
\hline & Realizar avaliação do programa de treinamento & TAPT [2.7] \\
\hline & Avaliar as dimensões de erros humanos & Erros humanos [4.1] \\
\hline \multirow{5}{*}{$\begin{array}{l}8 . \\
\text { Objetividade } \\
\text { e amplitude } \\
\text { do feedback }\end{array}$} & $\begin{array}{l}\text { Feedback imediato ao longo do treinamento e ao final (para } \\
\text { reforçar os acertos e corrigir os erros) }\end{array}$ & $\begin{array}{l}\text { Sistema de medição } \\
\text { de desempenho [2.6] }\end{array}$ \\
\hline & $\begin{array}{l}\text { Reforçar a motivação extrínseca com recompensas verbais ou } \\
\text { tangíveis, como por exemplo, pontuação e feedback positivo. }\end{array}$ & Motivação [3.1] \\
\hline & Atribuir sucessos e falhas ao jogador. & Confiança [3.2] \\
\hline & Reforçar o sucesso do aprendiz em completar uma tarefa. & Satisfação [3.2] \\
\hline & $\begin{array}{l}\text { Dar recompensas inesperadas, de modo que o jogador fique } \\
\text { satisfeito com o jogo, o treinamento realizado (desempenho } \\
\text { pessoal) e feedback fornecido. }\end{array}$ & Satisfação [3.2] \\
\hline
\end{tabular}

Esses critérios foram especificados e então utilizados na criação de um jogo sério (GLPSobControle), para treinamento e avaliação do controle de vazamento de gás de cozinha [Rocha e Campos, 2015], conforme descrito na próxima seção.

\section{Resultados}

O jogo GLPSobControle contem três fases $2 \mathrm{D}$ e três fases $3 \mathrm{D}$, é multiplataforma, com acesso on-line via navegador Web (Figura 1). Ao longo do processo de treinamento, há o uso de diferentes recursos (simulações $3 \mathrm{D}$, jogos simples $2 \mathrm{D}$, vídeos e um formulário de avaliação), conforme apresentados no Quadro 5.

Quadro 5. Fases do jogo GLPSobControle.

\begin{tabular}{|c|c|c|c|c|c|}
\hline \multicolumn{6}{|c|}{ Critérios Abordados } \\
\hline 2 & 3 & 6 & 7 & 7 & 8 \\
\hline Ref. & \multirow{2}{*}{$\begin{array}{c}\text { Níveis da } \\
\text { taxonomia de } \\
\text { Bloom } \\
\end{array}$} & \multirow{2}{*}{$\begin{array}{c}\text { Modos de aprendizagem } \\
\text { de Kolb e aprendizagem } \\
\text { experiencial }\end{array}$} & \multirow{2}{*}{$\begin{array}{c}\text { Tipos de } \\
\text { avaliação }\end{array}$} & \multirow{2}{*}{$\begin{array}{c}\text { Níveis de } \\
\text { avaliação de } \\
\text { Kirkpatrick } \\
\end{array}$} & \multirow{2}{*}{$\begin{array}{l}\text { Dimensões de } \\
\text { feedback }\end{array}$} \\
\hline (Fases) & & & & & \\
\hline (1) & 1: lembrar & $\begin{array}{c}\text { experiência concreta (fazer } \\
\text { e sentir) }\end{array}$ & diagnóstica & \multirow{6}{*}{ 2: aprendizado } & $\begin{array}{c}\text { construtivo e } \\
\text { descritivo }\end{array}$ \\
\hline (2) & 1: lembrar & $\begin{array}{l}\text { conceitualização abstrata } \\
\text { (pensar) }\end{array}$ & \multirow{3}{*}{ formativa } & & construtivo, \\
\hline (3) & 2: compreender & \multirow{2}{*}{$\begin{array}{l}\text { experiência ativa } \\
\text { (planejar e fazer) }\end{array}$} & & & prescritivo \\
\hline$(4)$ & 3: aplicar & & & & \\
\hline$(5)$ & 4: analisar & refletir a realidade & \multirow{2}{*}{ somativa } & & \multirow{2}{*}{$\begin{array}{c}\text { construtivo, e } \\
\text { descritivo }\end{array}$} \\
\hline$(6)$ & 5: sintetizar & (observar) & & & \\
\hline$(7)$ & 6: avaliar & - & autoavaliação & 1: reação & - \\
\hline
\end{tabular}


VI Congresso Brasileiro de Informática na Educação (CBIE 2017)

Anais do XXVIII Simpósio Brasileiro de Informática na Educação (SBIE 2017)

As fases (1) e (2) exploram o nível 1 da taxonomia de Bloom, com objetivos que possibilitam lembrar o protocolo treinado: (1) diagnosticar o conhecimento prévio por meio de clicar e arrastar cartas (Figura 1); e (2) relembrar ou aprender o protocolo básico, a partir das orientações das ações, que são ilustradas sequencialmente nas cartas 2D na parte superior da tela, e descritas na parte inferior (Figura 2). Há ainda orientações de cuidados a tomar durante o procedimento (cartas à esquerda).

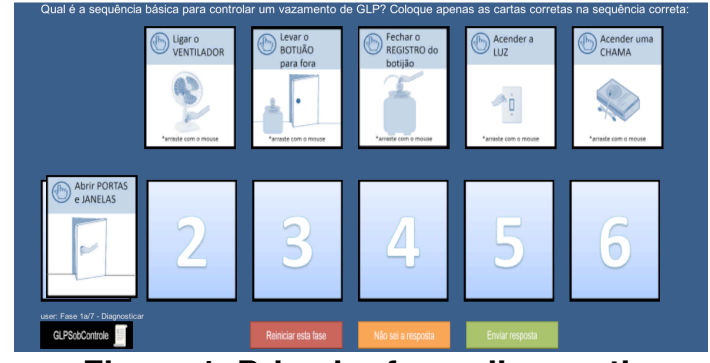

Figura 1. Primeira fase: diagnosticar.

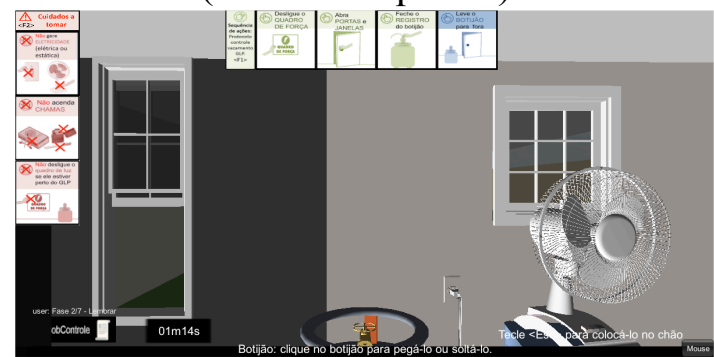

Figura 2. Segunda fase: lembrar.

O objetivo da terceira fase é verificar se o jogador compreendeu o uso do protocolo básico (mesmo cenário da fase 2, porém, sem orientações). A quarta fase possui um novo cenário 3D (Figura 3), com o objetivo de que o jogador aplique e treine o que foi aprendido sobre o uso do protocolo, sem orientações e com um novo desafio: salvar uma vítima que está próxima ao botijão de gás e ao quadro de força. Na quinta fase, o jogador assiste a um vídeo em que outra pessoa realiza o protocolo treinado. $\mathrm{O}$ objetivo é que ele analise as ações gravadas e identifique os erros realizados (Figura 4).

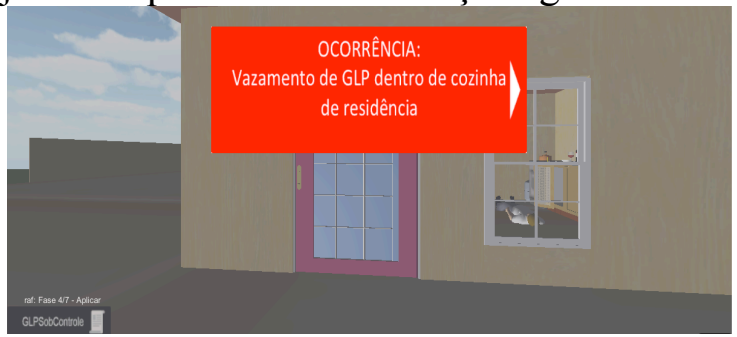

Figura 3. Quarta fase: aplicar.

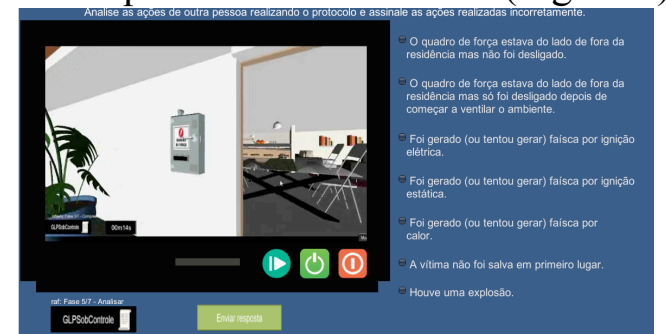

Figura 4. Quinta fase: analisar.

O objetivo da sexta fase é que o jogador sintetize o seu conhecimento combinando sentenças sobre o protocolo treinado. Para isto, ele arrasta as cartas para as casas inferiores, de modo a combinar a descrição de cada casa com a carta sobreposta. O objetivo da sétima e última fase é que o jogador se auto-avalie e avalie o jogo sério, por meio de um questionário validado por especialistas no domínio (Rocha, 2014). Todos os erros que podem ser realizados no treinamento são apresentados no Quadro 6.

As avaliações e validações do modelo de simulação de controle de vazamento de GLP, do jogo completo (conteúdo, avaliação e feedback) e do questionário, foram feitas por dois oficiais bombeiros; inclusive possibilitaram verificar uma oportunidade de melhoria no protocolo que não tinha sido observada apenas com o protocolo. Depois, os treinamentos foram realizados com uma amostra intencional de oito bombeiros (que abrange os perfis que utilizarão o jogo validado). Quanto a validade do conteúdo pelos aprendizes, ela foi realizada por meio do questionário e entrevistas abertas informais. Esta validação teve dois objetivos: (1) validar a representação física e comportamental da simulação criada (artefato final criado); e (2) verificar se os aprendizes conseguem relacionar o treinamento à realidade, por meio da autoavaliação. Após a realização do treinamento e coleta de dados (por meio do jogo sério e do questionário), os resultados foram organizados e analisados [Rocha, 2014][Rocha e Campos, 2015]. 
VI Congresso Brasileiro de Informática na Educação (CBIE 2017)

Anais do XXVIII Simpósio Brasileiro de Informática na Educação (SBIE 2017)

Quadro 6. Especificação dos erros humanos treinados e avaliados.

\begin{tabular}{|c|c|c|}
\hline Dimensões dos erros humanos & Erros & Consequências \\
\hline $\begin{array}{l}\text { Tempo: ação realizada cedo ou tarde } \\
\text { demais, ou omitida }\end{array}$ & $\begin{array}{l}\text { O bombeiro demora muito para salvar } \\
\text { uma vítima, ou não salva }\end{array}$ & $\begin{array}{l}\text { Morte de vítima por } \\
\text { asfixia }\end{array}$ \\
\hline $\begin{array}{l}\text { Distância: objeto ou controle foi } \\
\text { movido para perto ou longe demais }\end{array}$ & $\begin{array}{l}\text { A vítima foi removida para local inseguro } \\
\text { e não ventilado. } \\
\text { O botijão foi removido para um local não } \\
\text { ventilado }\end{array}$ & $\begin{array}{l}\text { Risco de asfixia (morte) } \\
\text { ou queimaduras (em } \\
\text { caso de incêndio) } \\
\text { Risco de explosão }\end{array}$ \\
\hline $\begin{array}{l}\text { Objeto: ação foi realizada com } \\
\text { objeto errado; no objeto errado } \\
\text { (parte ou todo); objeto pode estar } \\
\text { perto, ser similar ou não } \\
\end{array}$ & $\begin{array}{c}\text { O bombeiro gera energia elétrica (ascende } \\
\text { a luz, liga o ventilador), estática (corre } \\
\text { dentro da cozinha) ou por calor (liga o } \\
\text { isqueiro) }\end{array}$ & Explosão \\
\hline \multicolumn{3}{|c|}{ Sequência - erros na ordem das ações que podem variar: } \\
\hline $\begin{array}{l}\text {-Repetição: uma parte da sequência } \\
\text { é repetida por erro } \\
\end{array}$ & $\begin{array}{l}\text { O bombeiro desliga, liga, e desliga } \\
\text { novamente o registro de botijão }\end{array}$ & $\begin{array}{l}\text { Atraso no controle e } \\
\text { extinção do vazamento }\end{array}$ \\
\hline $\begin{array}{l}\text {-Omissão e esquecimento: uma parte } \\
\text { da sequência foi esquecida ou pulada }\end{array}$ & $\begin{array}{l}\text { O bombeiro retira o botijão da cozinha } \\
\text { antes de retirar a vítima }\end{array}$ & $\begin{array}{l}\text { Morte de vítima por } \\
\text { asfixia }\end{array}$ \\
\hline $\begin{array}{l}\text {-Inversão: duas partes da sequência } \\
\text { são trocadas }\end{array}$ & $\begin{array}{c}\text { O bombeiro retira a vítima antes de } \\
\text { desligar o botijão de gás }\end{array}$ & Risco de explosão \\
\hline -Ações erradas: & $\begin{array}{l}\text { O bombeiro desliga o quadro de força que } \\
\text { está dentro da cozinha próximo do gás } \\
\text { vazando }\end{array}$ & Explosão \\
\hline
\end{tabular}

\section{Discussões}

Por meio do uso de teorias e trabalhos das áreas de jogos, simulações, educação e treinamento, avaliação e domínio de aplicação, este trabalho propôs oito critérios que integra essas áreas visando a construção de jogos sérios efetivos.

Em relação ao critério "1. Relevância e fidelidade de cenários", foram usados os protocolos e manuais do bombeiro; abordadas as fidelidades físicas e funcionais (não abordou a psicológica), e analisado e incluído os erros humanos em procedimentos reais (Quadro 6). Apesar dos diferentes tipos de fidelidade serem importantes, é difícil mensurar a relação entre o grau de fidelidade e seus efeitos no treinamento e na educação (ambiente de aprendizagem), pois alguns estudos indicaram que a percepção de verossimilhança é mais importante, e que muitas vezes o alto realismo pode atrapalhar aprendizes novatos [Feinstein e Cannon, 2002]. Além de que, alguns componentes de simulação que reduzem o realismo podem aumentar o aprendizado, tais como, parar e reiniciar o modelo, ou um mecanismo refinado de feedback.

Quanto ao critério "2. Variedade de fases do programa de treinamento", e "3. Clareza e progressão dos objetivos e desafios" apenas o item "Adaptar as fases ao nível de competências prévias de cada aprendiz" não foi abordado, sendo que as fases estão descritas no Quadro 5. Porém, a adaptação pode ser realizada, em trabalhos futuros, a partir da avaliação do conhecimento prévio (realizada na fase 1). Por exemplo, um conteúdo oferecido no jogo poderia ser ignorado ou ser mais aprofundado.

Em relação aos critérios "4. Relevância e clareza de instruções e informações", "5. Clareza e simplicidade na interface e controle do jogo" e "6. Participação e independência do aprendiz", eles foram abrangidos também por meio do uso da taxonomia de Bloom, que permitiu criar as fases com desafios incrementais, e apresentá-las aos aprendizes durante a narrativa do jogo (objetivos, desafios, instruções, etc.), além da inclusão de diferentes atividades que engajam os aprendizes 
VI Congresso Brasileiro de Informática na Educação (CBIE 2017)

Anais do XXVIII Simpósio Brasileiro de Informática na Educação (SBIE 2017)

(principalmente, o ambiente 3D). Em relação à interface e controle, optou-se por colocar um tutorial padrão para todos, mas para usuários experientes ele poderia ser omitido, e para os iniciantes poderia haver uma fase para treinar os controles.

Os critérios "7. Objetividade e amplitude da avaliação" e "8. Objetividade e amplitude do feedback" foram contemplados no jogo sério, conforme Quadro 5. A motivação extrínseca foi reforçada com recompensas textuais (feedback positivo), porém, em trabalhos futuros, poderá ser incluída a pontuação e outros elementos de jogos que motivam o aprendizado.

Desta forma, não foram incluídos no jogo GLPSobControle os critérios de simular fidelidade psicológica, adaptar as fases ao nível de competências prévias do usuário, progredir as fases conforme o ritmo de cada aprendiz, reforçar a motivação com recompensas. Entretanto, esses critérios foram relatados pelos jogadores (nas respostas do questionário e nas entrevistas) como importantes e que sentiram falta.

\section{Considerações Finais}

Este trabalho se insere na pesquisa e prática em áreas que apoiam a criação de jogos sérios; de modo a entender como estes jogos devem ser planejados, desenvolvidos, avaliados e utilizados com o propósito de aprendizagem/ treinamento e avaliação do desempenho do aprendiz. A principal contribuição do conjunto de critérios criado é a integração de diferentes áreas para abranger os requisitos relacionados ao treinamento e avaliação do aprendiz por meio do uso do jogo sério. A falta de integração destas áreas, lacuna identificada nos trabalhos relacionados, foi o principal desafio pesquisado. Desta forma, os critérios listados neste artigo podem ser utilizados na fase de design do jogo do ciclo de vida de construção de jogos sérios e recursos digitais interativos (tais como, DevJSTA [Rocha, 2014] e AIMED [Rocha et al., 2017]).

O uso desses critérios auxiliou na criação de um jogo sério, sendo que foi possível planejar um balanceamento de jogabilidade, avaliação, simulação e conteúdo. Além disso, possibilitou a análise do que foi abordado ou não; sendo que as avaliações feitas pelos aprendizes corroboram para a necessidade dos critérios que não foram abordados no jogo sério criado (por exemplos, fidelidade psicológica, adaptar as fases ao nível de competências prévias, progredir as fases conforme o ritmo de cada aprendiz, reforçar a motivação com pontuações).

Como o jogo sério GLPSobControle tem indícios de possuir validade educacional [Rocha, 2014], então há indícios que esses critérios auxiliarão nas construções de jogos sérios balanceados (principalmente, jogabilidade e treinamento/ aprendizagem conforme planejado). Como trabalhos futuros, esses critérios serão usados na criação de jogos sérios de diferentes domínios de aplicação, além de aprimorar esse jogo criado.

\section{Referências}

ABNT (2001). NBR ISO 10015:2001- Gestão da Qualidade: diretrizes para treinamento. RJ: ABNT.

ADAMS, W.K.; et al. (2007). A Study of Educational Simulations Part I - Engagement and Learning. Journal of Interactive Learning Research, v. 12. pp. 397-419.

ALEXANDER; et al. From Gaming to Training: A Review of Studies on Fidelity, Immersion, Presence, and Buy-in and Their Effects on Transfer in PC-Based Simulations and Games. DARWARS Training Impact Group. 2005. 
VI Congresso Brasileiro de Informática na Educação (CBIE 2017)

Anais do XXVIII Simpósio Brasileiro de Informática na Educação (SBIE 2017)

ASLAN, S.; BALCI, O. (2015). GAMED: Digital Educational Game Development Methodology, In: Simulation, v. 91, n. 4, pp. 307-319.

BALCI, O. (2012). A Life Cycle for Modeling and Simulation. Simulation, v. 88, n. 7. pp. 870-883.

BANKS, J.; et al. (2001). Discrete-Event System Simulation. 3rd. ed. New Jersey: Prentice-Hall.

BECKER, K.; PARKER, J. (2012). Serious Instructional Design: ID for digital simulations and games. In: P. RESTA (ED.), Proceedings of Society for Information Technology \& Teacher Education International Conference 2012. Chesapeake, VA: AACE. pp. 2480-2485.

BLOOM, B.S. (1956). Taxonomy of Educational Objectives: the classification of educational goals - Handbook I: Cognitive Domain. New York: McKay.

CHANDLER, H.M. (2012). Manual de Produção de Jogos Digitais. 2. ed. Porto Alegre: Bookman.

DICK, W.; CAREY, L. CAREY, J.O. (2004). The Systematic Design of Instruction. 6th ed. Allyn \& Bacon.

FEINSTEIN, A. H.; CANNON, H. M. Constructs of Simulation Evaluation. Simulation \& Gaming, v. 33, n. 4, 2002. pp. 425-440.

FREITAS, S.; JARVIS, S. (2006). A Framework for Developing Serious Games to meet Learner Need. Interservice/Industry Training, Simulation, and Education Conference. 11p.

HAYS, R.T. (2005). The Effectiveness of Instructional Games: a literature review and discussion. Naval Air Warfare Center Training Systems Division (Technical Report).

HOLLNAGEL, E.; et al. (2011). Resilience Engineering in Practice: a guidebook. England: Ashgate.

IEEE. (2003). 1516.3-2003- IEEE Recommended Practice for High Level Architecture (HLA): federation development and execution process (FEDEP). New York: IEEE.

KIRKLEY, S.; TOMBLIN, S.; KIRKLEY, J. (2005). Instructional Design Authoring Support for the Development of Serious Games and Mixed Reality Training. I/ITSEC. Bloomington.

KIRKPATRICK, D.L.; KIRKPATRICK, J.D. (2006). Evaluating Training Programs: The Four Levels. 3rd Edition. San Francisco, CA, USA: Berrett-Koehle. 568 p.

KOLB, D.A. (1984). Experiential Learning: Experience as The Source of Learning and Development. Englewood Cliffs: Prentice-Hall Inc. pp. 20-38.

KORTELING, J.E., OPRINS, E.A.P.B.; KALLEN, V.L. (2012). Measurement of Effectiveness for Training Simulations. In: RTO System Analysis and Studies Panel (SAS), RTO-MP-SAS-095. Amsterdam, The Netherlands. pp. 1-14.

MARKLUND, B.B. (2013). Games in Formal Educational Settings Obstacles for the Development and Use of Learning Games. Licentiate Dissertation (Informatics), Univ. of Skövde, Sweden.

MILlER, A.; IMRIE, B.W.; COX, K. (1998). Functions of Assessment. In:__. Student Assessment in Higher Education: a handbook for assessing performance. London: Kogan Page. pp. 23-40.

NOVAK, J. (2010). Desenvolvimento de Games. São Paulo: Cengage. 443 p.

PISKURICH, G.; BECKSCHI P.; HALL, B. (2000). The ASTD Handbook of Training Design and Delivery: a comprehensive guide to creating and delivering training programs: instructor-led, computer-based, or self-directed. 2nd ed., New York: McGraw-Hill.

ROCHA, R.V. (2014). Metodologia Iterativa e Modelos Integradores para Desenvolvimento de Jogos Sérios de Treinamento e Avaliação de Desempenho Humano. Tese (Doutorado) Universidade Federal de São Carlos.

ROCHA, R.V.; CAMPOS, M.R. (2015), GLPSobControle: um jogo sério para o treinamento operacional de controle de vazamento de gás de cozinha. In: Apps.Edu (Protótipo) - Anais dos Workshops do IV Congresso Brasileiro de Informática na Educação, pp. 364-371.

ROCHA, RAFAELA V.; BITTENCOURT, I. I. ; ISOTANI, S. (2015). Análise, Projeto, Desenvolvimento e Avaliação de Jogos Sérios e Afins: uma revisão de desafios e oportunidades. In: XXVI Simpósio Brasileiro de Informática na Educação, pp. 692-701.

ROCHA, R.V.; et al. (2017). AIMED: Agile, Integrative and Open Method for Open Educational Resources Development. In: IEEE 17th International Conference on Advanced Learning Technologies (ICALT), Timisoara, Romania, pp. 163-167.

SALAS, E.; et al. (2009). Performance Measurement in Simulation-Based Training: A Review and Best Practices, In: Simulation Gaming, v. 40, n. 3, pp. 328-376.

SCHUYTEMA, P. (2008). Design de Games: uma abordagem prática. SP: Cengage Learning. 447 p. 